Conclusion The project demonstrates by empowering staff and building confidence, we have positively impacted on the choices available for patients and their families regarding the donation of tissues after death. Further education will be delivered to embed and ensure sustainability with this change in practice. A policy and procedure to standardise practice is being finalised

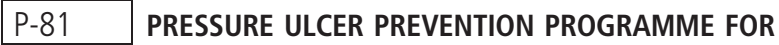 PALLIATIVE COMMUNITY PATIENTS - AN INNOVATION PROJECT}

Debra Boots, Nicola O'Shea, Julie Whiffin, Amanda Lawson, Nicola LePrevost. Pilgrims Hospices in East Kent, Margate, UK

\subsection{6/bmjspcare-2016-001245.104}

Background Skin integrity is key to ongoing care and comfort of patients towards end of life (Langemo, 2006). Palliative care aims to ease suffering with the raising prevalence of pressure area deterioration (EPUANPUAP, 2009). As patients' function and nutritional state declines, understanding early the risk of developing a pressure ulcer is pivotal. Prevention is key (NICE, 2015).

Funding, for a year-long project, was awarded by the Queen's Nursing Institute to a group of hospice community palliative nurses.

Project aims

- Explore early detection of risk factors in the development of pressure ulcers

- Understand the needs of patients and carers in the awareness of pressure ulcer risks

- Develop collaboration and sharing of knowledge with the Hospice multi-disciplinary team and develop relationships with primary care agencies.

Methods Three focus group meetings with patients and carers to discuss their pressure ulcer risk awareness and information needs.

Palliative care community nurses attended training. Meetings with external collaborative services, including community nurses, care agencies and care commissioning groups.

To compare the recording of Waterlow score before and after project implementation (Waterlow, 2005).

Results $94 \%$ of patients referred to the hospice by October 2015, had a Waterlow recording. Providing evidence for appropriate and timely pressure ulcer prevention interventions.

The focus group developed an information leaflet, 'Skin Awareness' advocating self-care as 'Awareness is Key'.

$100 \%$ of community based registered nurses received Waterlow Assessment training.

Awareness has been raised throughout the hospice multi-disciplinary team and external services (GPs, community curse, and occupational therapists) of pressure ulcer risks for community palliative care patients.

Conclusion The project has been vital in reducing the discomfort palliative care patients' experience towards end-of-life.

Further research is being developed, as there is a paucity of evidence in understanding the skin care of community palliative care patients, towards end-of life.

\section{P-82 THE UNPOPULAR PATIENTS IN PALLIATIVE CARE}

Maddy Bass. St Johns Hospice, Lancaster, UK

10.1136/bmjspcare-2016-001245.105
In 1972, Stockwell published her controversial research ("The unpopular patient") which described nurses' relationships with patients who they perceived as "difficult", and focussed on negative stereotyping. This was based around nurses giving preferential care to favoured patients.

Does this exist in palliative care, which prides itself on gold standard and person-centred care, across all care settings and particularly in specialist palliative care units and hospices?

During the author's work in various palliative care settings and varying roles, it has been evident through a variety of approaches that even in palliative care patients can still be perceived as "unpopular". This can, in turn, exacerbate that patient's unpopular behaviour further (Goffman, 1963).

Dame Cicely Saunders stated, "You matter because you are you. We will do all we can to help you live until you die". Equitable care access should prevent stereotyping, however the question is, do staff still label patients and families as unpopular? Is this simply an attitude and opinion? Does it affect the care they receive?

A brief literature review explores the concept of the unpopular patient in various care settings but not in palliative care. This work will complete a detailed literature review of "the unpopular patient”, the principles of palliative care and equitable access, and will be presented at the conference.

In future, research is required: initially this will be small scale through questionnaires to staff in a variety of palliative care settings and roles, using methodology similar to the original research. Following this, focus groups will be held to explore the emerging themes in more detail, and possibly individual interviews may be held with specific staff for richer data.

It is hoped that publishing and sharing this work will make staff working in palliative care self-aware about these attitudes.

\section{P-83 \\ FROM CRITICAL INCIDENT TO IMPROVED DOCUMENTATION; A MULTI-DISCIPLINARY APPROACH}

Lorraine West, Mark Harrington. St. Luke's Hospice, Sheffield, UK

\subsection{6/bmjspcare-2016-001245.106}

Background An inpatient developed a grade 4 pressure ulcer, however, inadequate documentation demonstrating the on-going management and escalation of the incident made it difficult to answer both the patient and their family's questions. The incident was escalated to the relevant bodies and an internal investigation undertaken. The hospice and the patient's family were keen to ensure lessons were learnt and that changes were made to improve documentation.

Aim To develop an effective process and training programme ensuring comprehensive documentation of patient care.

Methods Mandated documentation training focusing on clinical standards and the implications of poor record keeping was jointly developed with Her Majesty's Coroner and delivered at the Coroner's Court. A Clinical Documentation Management group was developed at the hospice to provide strategic direction and control.

A multi-disciplinary audit tool was developed to evaluate patient records and a six-monthly audit cycle established. Real time feedback highlighting positive practices and areas for improvement was provided to managers and clinicians. Results of the audit indicated changes were required within the documentation process; all paper assessment forms were reviewed and a 